\title{
Article \\ Chitin Glucan Shifts Luminal and Mucosal Microbial Communities, Improve Epithelial Barrier and Modulates Cytokine Production In Vitro
}

\author{
Marta Calatayud ${ }^{1,2, *,+}$, Lynn Verstrepen ${ }^{1,+}$, Jonas Ghyselinck ${ }^{1}$, Pieter Van den Abbeele ${ }^{1}$, Massimo Marzorati ${ }^{1,2, *}$, \\ Salvatore Modica $^{3}$, Thibaut Ranjanoro ${ }^{3}$ and Véronique Maquet ${ }^{3}$ \\ 1 ProDigest BV, Technologiepark 82, 9052 Ghent, Belgium; Lynn.Verstrepen@prodigest.eu (L.V.); \\ Jonas.Ghyselinck@prodigest.eu (J.G.); pieter.vandenabbeele@telenet.be (P.V.d.A.) \\ 2 Center for Microbial Ecology and Technology (CMET), Ghent University, Coupure Links 653, \\ 9052 Ghent, Belgium \\ 3 KitoZyme SA., Parc Industriel des Hauts Sarts, 4040 Herstal, Belgium; s.modica@kitozyme.com (S.M.); \\ t.ranjanoro@kitozyme.com (T.R.); v.maquet@kitozyme.com (V.M.) \\ * Correspondence: marta.calatayud@prodigest.eu (M.C.); Massimo.Marzorati@prodigest.eu or \\ Massimo.marzorati@ugent.be (M.M.) \\ + These authors contributed equally to the work.
}

\section{check for} updates

Citation: Calatayud, M.; Verstrepen, L.; Ghyselinck, J.; Van den Abbeele, P.; Marzorati, M.; Modica, S.; Ranjanoro, T.; Maquet, V. Chitin Glucan Shifts Luminal and Mucosal Microbial Communities, Improve Epithelial Barrier and Modulates Cytokine Production In Vitro. Nutrients 2021, 13, 3249. https://doi.org/10.3390/ nu13093249

Academic Editor: Yoshitaka Hashimoto

Received: 9 August 2021

Accepted: 17 September 2021

Published: 18 September 2021

Publisher's Note: MDPI stays neutral with regard to jurisdictional claims in published maps and institutional affiliations.

Copyright: (c) 2021 by the authors. Licensee MDPI, Basel, Switzerland. This article is an open access article distributed under the terms and conditions of the Creative Commons Attribution (CC BY) license (https:// creativecommons.org/licenses/by/ $4.0 /)$

\begin{abstract}
The human gut microbiota has been linked to the health status of the host. Modulation of human gut microbiota through pro- and prebiotic interventions has yielded promising results; however, the effect of novel prebiotics, such as chitin-glucan, on gut microbiota-host interplay is still not fully characterized. We assessed the effect of chitin-glucan (CG) and chitin-glucan plus Bifidobacterium breve (CGB) on human gut microbiota from the luminal and mucosal environments in vitro. Further, we tested the effect of filter-sterilized fecal supernatants from CG and CGB fermentation for protective effects on inflammation-induced barrier disruption and cytokine production using a co-culture of enterocytes and macrophage-like cells. Overall, CG and CGB promote health-beneficial short-chain fatty acid production and shift human gut microbiota composition, with a consistent effect increasing Roseburia spp. and butyrate producing-bacteria. In two of three donors, CG and CGB also stimulated Faecalibacterium prausniitzi. Specific colonization of B. breve was observed in the lumen and mucosal compartment; however, no synergy was detected for different endpoints when comparing CGB and CG. Both treatments included a significant improvement of inflammationdisrupted epithelial barrier and shifts on cytokine production, especially by consistent increase in the immunomodulatory cytokines IL10 and IL6.
\end{abstract}

Keywords: chitin-glucan; prebiotic; gut microbiome; intestinal barrier; immunomodulation

\section{Introduction}

During the past few years, published reports have suggested that gut microbiota of the host has been linked to the health status of an individual. The largest pool of microbes occurs in the distal gastrointestinal tract, both in the lumen, where metabolic potency of the microbial ecosystem allows for the fermentation of non-digestible carbohydrates, xenobiotic metabolism, and vitamin and neuropeptide production, as well as in the mucosal niche, where specific microbial communities have a close interaction with host cells [1]. One of the main factors shaping gut microbial communities is the diet. Specifically, dietary fibers are actively fermented by gut microbes leading to the production of short-chain fatty acids [2]. Dietary fiber is composed of carbohydrate polymers undigested and unabsorbed in the human small intestine [3-5], including resistant oligosaccharides, nonstarch polysaccharides, resistant starches, and lignin [6].

Prebiotics are defined as substrates selectively utilized by host microorganisms, conferring a health benefit [7]. Symbiotics are classified as complementary symbiotics, which 
must be composed of a probiotic plus a prebiotic, and synergistic symbiotics, for which the substrate is designed to be selectively utilized by the co-administered microorganisms [8]. The beneficial effects of dietary fiber intake on human health have been described, such as a reduced risk for heart disease, stroke, hypertension, specific gastrointestinal disorders, obesity, type 2 diabetes, and some cancers $[9,10]$. These effects are potentially related to the modulation of the gut microbiota and host metabolism [11]. Although the adequate intake of fiber is associated with many health benefits $(25 \mathrm{~g} / \mathrm{d}$ for healthy adults, European Food Safety Agency-EFSA) [12], the European and USA population is still below recommended levels [6,9].

In this context, dietary supplementation with prebiotic products can be an approach to cover the gap in fiber intake and promote eubiotic gut microbial communities. At the same time, a symbiotic intervention would also benefit the consumer from the complementary and synergistic effect of both pre- and probiotic elements.

The most studied prebiotics are a subset of carbohydrate groups, mainly oligosaccharides [13]. Incorporating novel fibers into the diet can diversify the source of prebiotics for human consumption, also adding value to food industry. In that sense, chitinglucan is an insoluble fiber composed of chitin ( $\beta-1,4-$ poly-N-acetyl-D-glucosamine) and $\beta$-1,3-D-glucan, obtained from Aspergillus niger mycelial cell walls, and it is considered a safe food ingredient by the EFSA, with a recommended dose of up to $5 \mathrm{~g} /$ day [14].

Human intervention studies have shown the potential health benefits of chitin-glucan intake. Daily consumption of $4.5 \mathrm{~g}$ of CG during 6 weeks was associated with reduced oxidized LDL blood levels in a randomized, double-blind, placebo-controlled study $(n=130)$ [15]. Recently, changes in gut microbiota and derived metabolites have been described in 15 healthy human volunteers consuming $4.5 \mathrm{~g} /$ day of CG (3 weeks) [16]. Authors described a specific increase in Roseburia and Eubacterium genera associated with CG consumption, supporting the results obtained in in vitro and pre-clinical animal models $[17,18]$.

However, the effect of chitin-glucan on the gut mucosal environment and on the hostmicrobiota interplay, and the potential synergistic effect of chitin-glucan with a probiotic strain has not yet been evaluated.

In this research, we assessed the effect of chitin-glucan (CG) and chitin-glucan plus Bifidobacterium breve (CGB) on gut microbiota structure and function, using a colonic fermentation in vitro model simulating the colonic luminal and mucosal compartments of three healthy donors. Supernatants obtained from CG and CGB treatments were subsequently applied to a gut inflammation model of Caco-2 and THP-1 cells to assess the host-microbiota interplay.

\section{Materials and Methods}

\subsection{Short-Term Colonic Incubations}

The objective of these experiments was to determine the effect of chitin-glucan and chitin-glucan and B. breve supplementation in vitro on the gut microbial community structure and function from three healthy individuals. The combination of chitin-glucan with B. breve was included to compare the effect of chitin-glucan alone (prebiotic) and the potential synergism of a prebiotic and a probiotic strain (symbiotic). Previous literature have shown that $B$. breve can improve the endoscopic scores of inflammatory bowel patients, suggesting a role in immunoregulation [19]. In addition, $B$. breve has potential to reduce body fat in healthy pre-obese individuals [20].

Short-term colonic incubations were performed as previously described in Van den Abbeele et al., [21] with some modifications. Briefly, fresh fecal material (29-35 y, 1 male, 2 females) was collected and independently homogenized in anaerobic phosphate buffer $\left(\mathrm{K}_{2} \mathrm{HPO}_{4} 8.8 \mathrm{~g} / \mathrm{L} ; \mathrm{KH}_{2} \mathrm{PO}_{4} 6.8 \mathrm{~g} / \mathrm{L}\right.$; sodium thioglycolate $0.1 \mathrm{~g} / \mathrm{L}$; sodium dithionite $0.015 \mathrm{~g} / \mathrm{L})$ (Chem-lab NV, Zedelgem, Belgium) $(1: 5 \mathrm{w} / \mathrm{v})$ using a stomacher bag mixer 10 min (BagMixer 400, Interscience, Louvain-LaNeuve, Belgium). Big particles were removed by centrifugation ( $2 \mathrm{~min}, 500 \mathrm{~g})$, and the fecal slurries $(10 \% \mathrm{v} / \mathrm{v})$ were inoculated in colonic reactors containing CG and CGB and anaerobic sugar-depleted nutritional medium 
(3.5 g/L K ${ }_{2} \mathrm{HPO}_{4}, 10.9 \mathrm{~g} / \mathrm{L} \mathrm{KH}_{2} \mathrm{PO}_{4}, 2 \mathrm{~g} / \mathrm{L} \mathrm{NaHCO} 3$ (Chem-lab NV, Zedelgem, Belgium), $2 \mathrm{~g} / \mathrm{L}$ Yeast Extract, $2 \mathrm{~g} / \mathrm{L}$ peptone (Oxoid, Aalst, Belgium), $0.5 \mathrm{~g} / \mathrm{L} \mathrm{L}$-cysteine and $2 \mathrm{~mL} / \mathrm{L}$ Tween80 (Sigma-Aldrich, Bornem, Belgium)) at pH 6.5. In addition, colonic bioreactors included five mucin-coated carriers, prepared according to Van den Abbeele et al., 2013 [22]. Briefly, K1-carriers (AnoxKaldnes AB, Lund, Sweden) were submerged in a mucin dissolution composed by $0.5 \mathrm{~g} / \mathrm{L}$ gastric porcine mucin type II (Sigma-Aldrich, Bornem, Belgium) and $0.1 \mathrm{~g} / \mathrm{L}$ bacteriological agar (Oxoid, Aalst, Belgium), combined in a polyethylene netting (Zakkencentrale, Rotterdam, The Netherlands) and submerged in the colonic simulated media.

For each donor, a control condition, a single dose of $5 \mathrm{~g} / \mathrm{L}$ chitin-glucan (CG/prebiotic), or a single dose of $5 \mathrm{~g} / \mathrm{L}$ chitin-glucan + B. breve $\left(1.4 \times 10^{7}\right.$ colony forming units) (CGB/symbiotic) were tested. CG and CGB were obtained from Kitozyme, Herstal, Belgium. B. breve included in CGB was obtained from THT (Gembloux, Belgium). The dose applied in this study was based on the intended intake of CG described as safe by the EFSA [14], and considering the volume of the colon $(1 \mathrm{~L})$ of healthy individuals [23].

Incubations were performed in anaerobic conditions for $48 \mathrm{~h}$ at $37^{\circ} \mathrm{C}, 90 \mathrm{rpm}$ and samples were collected at different time points to analyze markers of microbial activity $(0,6,24$, and $48 \mathrm{~h}$ ) and composition (qPCR of bifidobacteria and Faecalibacterium prausnitzii and 16S-targeted Illumina sequencing; 0 and $48 \mathrm{~h}$ ).

\subsection{Microbial Metabolic Activity: pH, Gas Production, Short-Chain Fatty Acids (SCFA) and Ammonium}

Metabolic activity of the gut microbial communities was assessed by quantifying general markers of fermentation ( $\mathrm{pH}$ and gas production), lactate and short chain fatty acids.

The $\mathrm{pH}$ (Senseline F410; ProSense, Oosterhout, The Netherlands), gas (hand-held pressure indicator CPH6200; Wika, Echt, The Netherlands), lactate (LA), and short-chain fatty acid (SCFA) measurements were performed at $0,6,24$, and $48 \mathrm{~h}$ after starting the colonic incubation. Acetate, propionate, butyrate, and branched SCFAs (isobutyrate, isovalerate, and isocaproate) were measured as described by De Weirdt et al., [24]. According to the manufacturer's instructions, lactate quantification was performed using a commercial enzymatic assay kit (R-Biopharm, Darmstadt, Germany).

\subsection{DNA Extraction and $16 S$ RNA Sequencing}

DNA was isolated starting from cell pellets from $1 \mathrm{~mL}$ sample aliquots (luminal compartment) or $0.25 \mathrm{~g}$ sample (mucosal compartment) at 0 and $48 \mathrm{~h}$. Bacterial cells

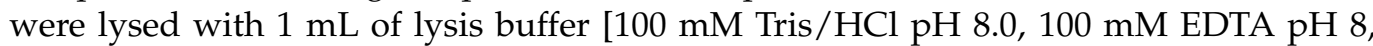
$100 \mathrm{mM} \mathrm{NaCl}, 1 \%(\mathrm{~m} / \mathrm{v})$ polyvinylpyrrolidone and $2 \%(\mathrm{~m} / \mathrm{v})$ sodium dodecyl sulphate] and $200 \mathrm{mg}$ of glass beads $\left(0.11 \mathrm{~mm}\right.$, Sartorius, Schaerbeek, Belgium), in a FastPrep ${ }^{\circledR}$ 96 instrument (MP Biomedicals, Santa Ana, CA, USA), two times for $40 \mathrm{~s}$ at $1600 \mathrm{rpm}$. After removing glass beads ( $5 \mathrm{~min}, 13,000 \times g)$, DNA was extracted following a phenolchloroform extraction. DNA was precipitated with 1 volume ice-cold isopropyl alcohol and 0.1 volume $3 \mathrm{M}$ sodium acetate $\left(1 \mathrm{~h},-20^{\circ} \mathrm{C}\right)$. DNA pellet was dried and resuspended in $100 \mu \mathrm{L} 1 \times$ TE $(10 \mathrm{mM}$ Tris, $1 \mathrm{mM}$ EDTA) buffer. Samples were diluted in DNAse/RNAse free water to obtain final concentrations of $50 \mathrm{ng} / \mu \mathrm{L}$ and stored at $-20^{\circ} \mathrm{C}$. Additionally,16S rRNA gene amplicon sequencing of the V3-V4 region was analyzed at LGC Genomics (Teddington, Middlesex, UK). The 341F (5'-CCTACGGGNGGCWGCAG-3') and 785R (5'-GACTACHVGGGTATCTAAKCC-3') primers were used according to [25]. Quality control PCR was conducted using Taq DNA Polymerase with the Fermentas PCR Kit according to the manufacturers' instructions (Thermo Fisher Scientific, Waltham, MA, USA). The DNA quality was verified by electrophoresis on a $2 \%(w / v)$ agarose gel for $30 \mathrm{~min}$ at 100 V. 2.8 and selective quantification of double strand DNA BY Qubit dsDNA High Sensitivity Assay Kit, following manufacturer instructions. 


\subsection{Bioinformatics Analysis of Amplicon Data}

The mothur software package (v. 1.39.5) and guidelines were used to process the amplicon data generated by LGC Genomics as previously described in De Paepe et al., 2017 [25]. An operational taxonomic unit (OTU) was defined as a collection of sequences with a length between 402 and 427 nucleotides that are found to be more than $97 \%$ similar to one another in the V3-V4 region of their 16S rRNA gene after applying Opticlust clustering [26-29]. Taxonomy was assigned using the RDP version 16 and silva.nr_v123 database [30-32]. The shared file, containing the number of reads observed for each OTU in each sample, was loaded into Microsoft ${ }^{\circledR}$ Excel $^{\circledR} 2016$ MSO (16.0.11901.20070, Redmond, WA, USA). Reads occurring only 5 times in all samples were removed, as they were supposedly artifacts or bacteria that were not having any biological impact. For the most abundant OTUs, the sequences retrieved from a 3\% dissimilarity level fasta file obtained in mothur were classified through the RDP web interface using the RDP SeqMatch tool. The database search was restricted to type strains with only near-full-length and good quality sequences. The sequences were blasted in NCBI against the $16 \mathrm{~S}$ rRNA gene sequences, selecting only type material, with optimization of the BLAST algorithm for highly similar sequences (accession date: December 2018) [30,32]. Although identification to the species level based on short $300 \mathrm{bp}$ reads may involve some ambiguity, the most likely species classification of a few interesting OTUs is reported in the results sections. In the event of inconsistencies in the results of the RDP SeqMatch tool and NCBI BLAST, no species-level classification is provided.

\subsection{Microbial Community Analysis by $q P C R$}

qPCR was used to verify microbial changes detected by $16 \mathrm{~S}$ rRNA sequencing on specific taxa.

Samples collected after 0 and $48 \mathrm{~h}$ of incubation were evaluated for the total amount of bifidobacteria and F. prausnitzii by qPCR. The DNA was extracted as described in Section 2.3. qPCR assays were performed using a StepOnePlus Real-Time PCR system (Applied Biosystems, Foster City, CA, USA), using the primers and conditions described in Supplementary Tables S1 and S2, adapted from Rinttilä et al., 2004 and Lopez-Siles et al., 2014 [33,34]. Bioline qPCR master mix was obtained from GC Biotech B.V. (Belgium). Each sample was analyzed in technical triplicates and outliers (more than $1 \mathrm{CT}$ difference) were omitted. The samples were checked for correct melt curve peaks. The standard curves for all of the different runs had efficiencies between 90 and $105 \%$. Results are reported as logs (16S rRNA gene copies/mL).

\subsection{Flow Cytometry}

For flow cytometry analysis, 10 -fold serial dilutions of luminal and mucosal samples were prepared in anaerobic Dulbecco's Phosphate-buffered Saline (DPBS) (Sigma-Aldrich, Bornem, Belgium) and stained with $0.01 \mathrm{mM}$ SYTO24 (Life Technologies Europe, Merelbeke, Belgium) for $15^{\prime}$ at $37^{\circ} \mathrm{C}$ in the dark. Samples were analyzed on a BD Facsverse (BDBiosciences, Erembodegem, Belgium) using the high-flow-rate setting. Bacteria were separated from medium debris and signal noise by applying a threshold level of 200 on the SYTO channel. Flow cytometry data were analyzed using FlowJo, version 10.5.2.

\subsection{Cell Culture}

Caco-2 cells (HTB-37; American Type Culture Collection) were maintained in Dulbecco's modified eagle medium (DMEM) containing glucose and glutamine and supplemented with HEPES and 20\% $(v / v)$ heat-inactivated (HI) fetal bovine serum (FBS). THP1-Blue ${ }^{\mathrm{TM}}$ NF- $\mathrm{kB}$ reporter cells were obtained from InvivoGen (Toulouse, France) and maintained in Roswell Park Memorial Institute (RPMI) 1640 medium containing glucose $(2 \mathrm{~g} / \mathrm{L})$ and glutamine $(0.3 \mathrm{~g} / \mathrm{L})$, supplemented with HEPES $(0.1 \mathrm{mM})$, sodium pyruvate $(1 \mathrm{mM})$ and $10 \%(v / v)$ HI-FBS. Cells were incubated at $37^{\circ} \mathrm{C}$ in a humidified atmosphere of air $/ \mathrm{CO}_{2}(95: 5, v / v)$. 
The Caco-2/THP-1 co-culture was performed as previously described [35]. Briefly, Caco-2 monolayers were cultured for 14 days on 24-well semi-permeable supports, until a functional cell monolayer was obtained. Then, $48 \mathrm{~h}$ before the start of the co-culture, THP1-Blue ${ }^{\mathrm{TM}}$ cells were seeded in 24-well plates and stimulated for $48 \mathrm{~h}$ with phorbol 12-myristate 13-acetate (PMA; $100 \mathrm{nM}$ ). After PMA removal, Caco-2 were placed on top of PMA-differentiated THP1-Blue ${ }^{\mathrm{TM}}$ cells, followed by apical treatment with complete medium or filter-sterilized $(0.22 \mu \mathrm{m})$ colonic suspensions collected after $24 \mathrm{~h}$ and $48 \mathrm{~h}$ of incubation. All treatments were done in biological triplicate. Transepithelial electrical resistance (TEER) was assessed at $0 \mathrm{~h}$ and after $24 \mathrm{~h}$ of co-culturing, as estimation of the integrity of the epithelial barrier. Further, after $24 \mathrm{~h}$, basolateral medium was removed and THP1Blue $^{\mathrm{TM}}$ were stimulated with ultrapure lipopolysaccharide (LPS) from Escherichia coli K12 (InvivoGen, Toulouse, France; $10 \mathrm{ng} / \mathrm{mL}$ ) or left untreated. After $6 \mathrm{~h}$, basolateral medium was collected for cytokine (Luminex technology, 's-Hertogenbosch, The Netherlands ) and NF-kB measurements (QUANTI-Blue reagent, InvivoGen, Toulouse, France).

\subsection{Data Processing and Statistics}

All statistical analyses were performed in GraphPad Prism version 8.2.0 for Windows (GraphPad Software, San Diego, CA, USA). All formal hypothesis tests were conducted on the $5 \%$ significance level $(\alpha=0.05)$. Metabolic data, including $\mathrm{pH}$, gas, SCFAs, BCFAs, and lactate were reported as the change between 6 and $0 \mathrm{~h}(\Delta 6 \mathrm{~h}), 24$ and $6 \mathrm{~h}(\Delta 24 \mathrm{~h})$, and 48 and $24 \mathrm{~h}(\Delta 48 \mathrm{~h})$. Descriptive statistics (arithmetic mean and standard error of the mean (SEM)) for all treatment groups were used to summarize the outcomes. Non-paired $t$-tests were used for comparisons of treatments, with Holm-Š́ídák test for post hoc pairwise comparisons of treatments and control condition.

Then, 16S RNA data (i.e., proportional abundances (\%) were multiplied by the absolute cell numbers (cells $/ \mathrm{mL}$ ) obtained via flowcytometry to obtain quantitative data at phylum, family and OTU level. To establish a LOQ for quantitative 16S-targeted Illumina sequencing data, one read was divided by the total amount of reads in each sample, followed by multiplication with the bacterial cell count detected by flow cytometry. This allowed to obtain a LOQ for each sample individually. Quantitative data was analyzed using Calypso software version 8.84 , and specific tests were described in the corresponding section.

For cell culture assays, treatment samples were compared to the control samples using two-way ANOVA with Sidak's multiple comparisons test. Significant differences are represented by $\left({ }^{*}\right) .\left({ }^{*}\right),(* *),\left({ }^{* * *}\right)$, and $\left({ }^{* * *}\right)$ represent $p<0.05, p<0.01, p<0.001$, and $p<0.0001$, respectively.

\section{Results}

\subsection{Chitin-Glucan and Chitin-Glucan + B. breve Affect Fermentation Markers and Induce} SCFA Production

General markers of microbial fermentation ( $\mathrm{pH}$ decrease and gas production) were increased in CG- and CGB-treated reactors compared to the control condition (Figure 1A,B). In general, CG- and CGB-treated reactors showed an increase in SCFAs at $48 \mathrm{~h}(\Delta 48 \mathrm{~h}$; $p<0.05$ ), with fold changes to control of approximately 1.6, 2, 3 and 1.7 for acetate, propionate, butyrate, and total SCFA, respectively (Figure 1C-F). Overall, propionate and butyrate did not show significant differences between CG and CGB treatments (Supplementary Figure S1). 


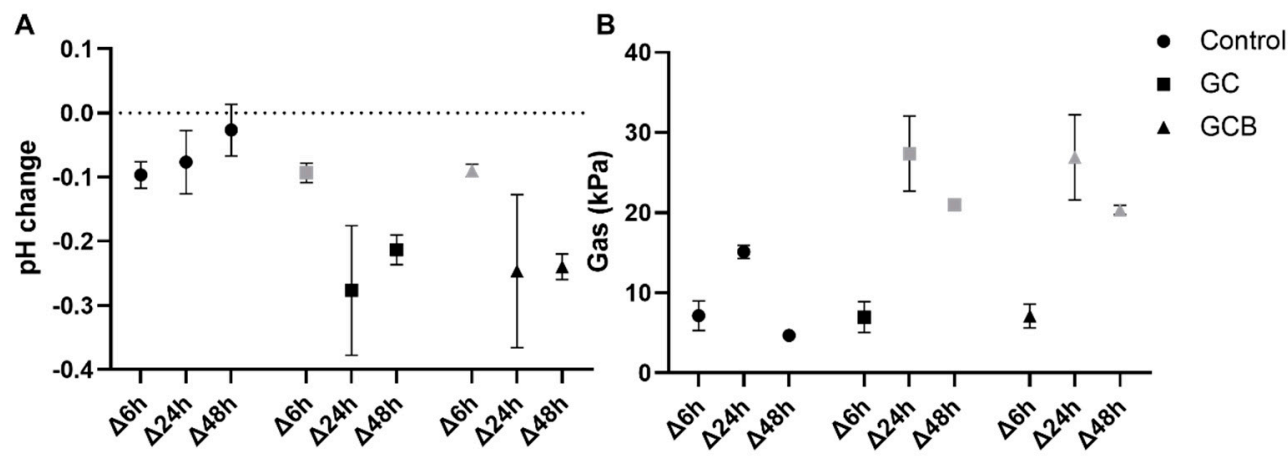

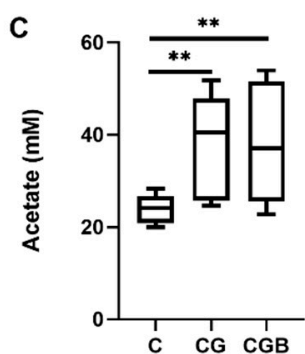

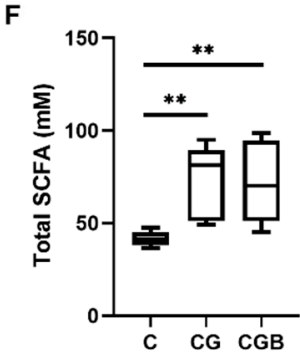

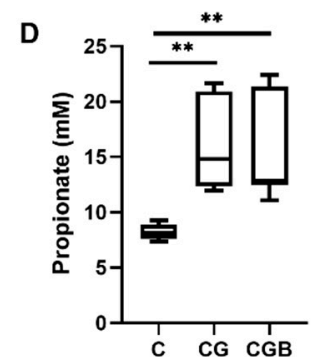

G

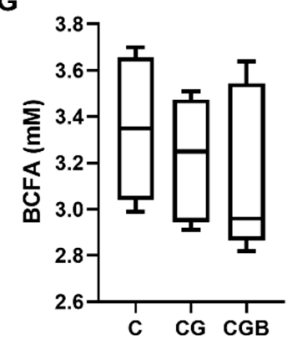

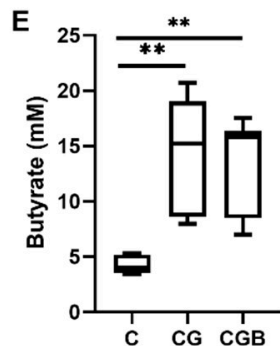

H

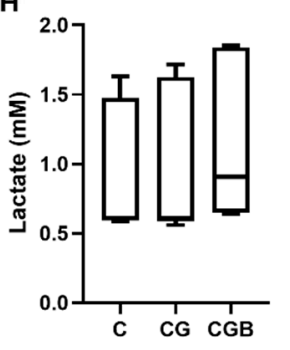

Figure 1. Effect of chitin-glucan and chitin-glucan + B. breve on markers of gut microbial metabolism. (A) Gas and (B) pH change at different time intervals; (C-E) represent box plots of acetate, propionate, butyrate, total SCFA, BCFA $(\triangle 48 \mathrm{~h})$, and lactate $(\Delta 6 \mathrm{~h})$ change for different treatments. $(\mathbf{A}, \mathbf{B})$ graphs represent mean $\pm \mathrm{SEM}, \mathrm{n}=$ three donors. Box plots represent data from $\mathrm{n}=$ three donors with the line at the mean. Statistically significant differences $(p<0.05)$ between treatments and controls are marked with grey symbols in $(\mathbf{A}, \mathbf{B})$ and with asterisks $\left({ }^{* *} p<0.01\right)$ in $(\mathbf{C}-\mathbf{H})$.

When analyzing each donor independently, donors 1 and 3 had the highest response to CG and CGB in total SCFA production (two-fold change to control), with the highest butyrate production observed in donor 1 (CG $=4.8 \pm 0.4$ and $C G B=4.1 \pm 0.3$-fold change to control) and the highest propionate increase shown in donor 3 (CG $=2.7 \pm 0.1$ and $\mathrm{CGB}=2.8 \pm 0.1$-fold change to control). Overall, donor 2 was the least responsive to CG and CGB.

BCFA and lactate showed high interindividual variability, and when considering the three donors together, no significant differences were observed compared to control (Figure 1G,H). When looking at each donor, lactate increased compared to the control in the CGB-exposed group $(\Delta 6 \mathrm{~h}, p<0.05)$ (Supplementary Table S3), with donor 1 showing the highest level. BCFAs were reduced in donor 2 and 3 CG-treated reactors and CGB-treated reactors (Supplementary Table S4).

\subsection{CG and CGB Modify Microbiota Structure at the Luminal and Mucosal Level}

The impact of CG and CGB supplementation on gut microbial structure was analyzed by comparing treated reactors to control reactors containing the same background nutritional media without any treatment.

In the luminal compartment, CG and CGB increased Chao1 index $(p=0.044)$

(Supplementary Figure S2), whereas in the mucosal compartment, no significant effects were observed for alpha diversity indices, richness or evenness (Supplementary Figure S3). 
At family level, a discriminant analysis of principal components (DAPC) showed a different clustering of CG and CGB to control ( $p=0.008$, Adonis test based on Bray-Curtis distance) (Figure 2A). To determine which features could explain the differences between clusters, we used linear discriminant analysis of effect size (LEfSe). LEfSe analysis showed an enrichment of Butyriciococcaceae in CGB-treated reactors and Clostridiaceae, Tannerellaceae and Erysipelatoclostridiaceae in CG-treated reactors (LDA score > 3, Figure 2B).

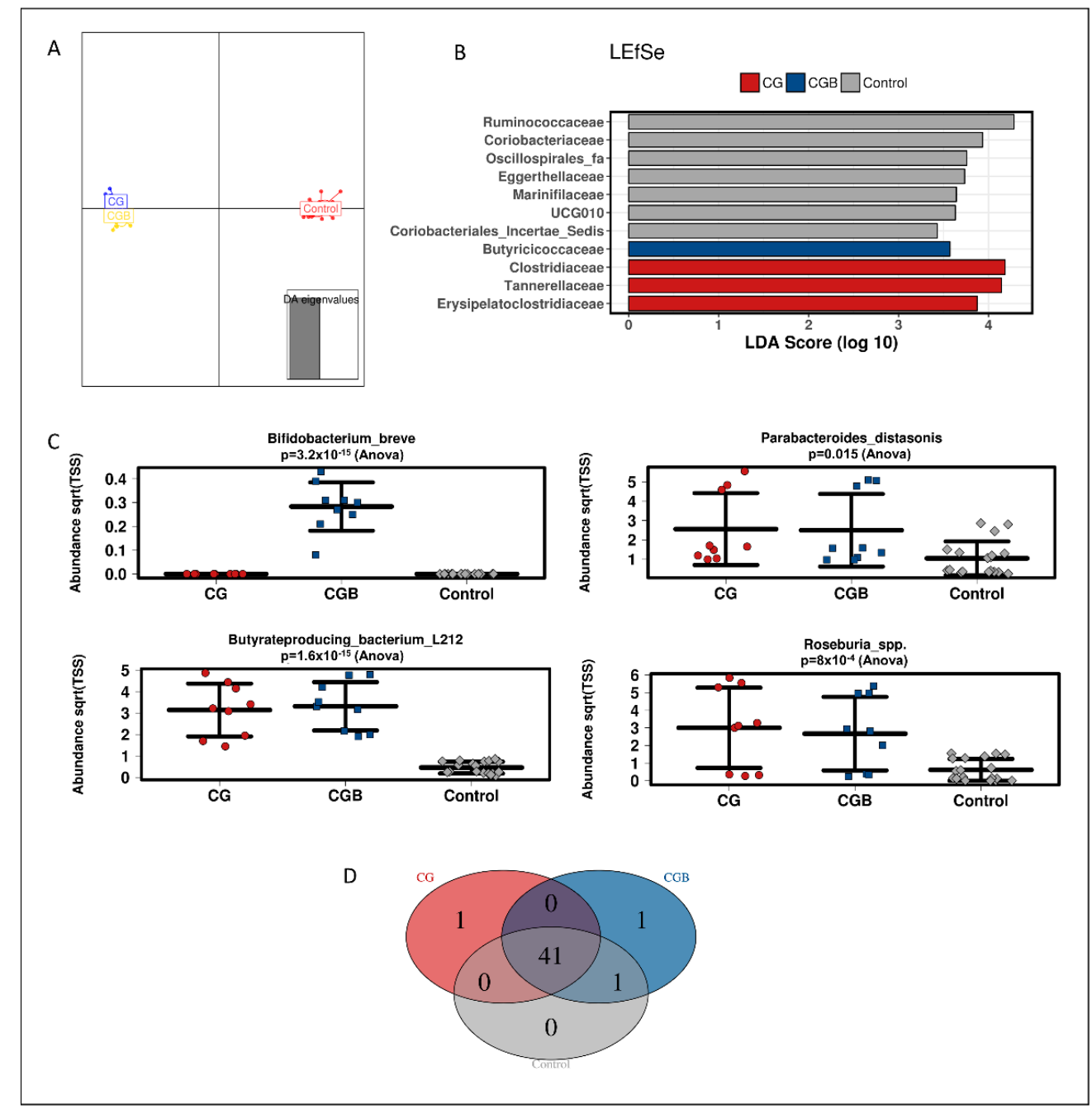

Figure 2. Effect of GC and GCB treatments on luminal microbial composition. (A) Discriminant analysis of principal components (DAPC) plot of the luminal compartment at family level; (B) linear discriminant analysis effect size (LEfSe) at family level; (C) strip charts of selected features at OTU level; (D) core microbiota at OTU level. Graphs represent 16S rRNA sequencing data from three healthy individuals $(\mathrm{n}=3)$ in technical triplicates $(\mathrm{n}=3)$.

At OTU level, Butyrate-producing bacterium-L212 (OTU7), Parabacteroides diastonis (OTU12/45) and Roseburia spp. (R. hominis and R. inulinivorans; OTU1/OTU2) were enriched by CG and CGB (Figure 2C). B. breve (related to OTU211) was increased by CGB treatment. B. breve was a unique feature for the CGB treatment (Figure 2D), indicating the absence of this specific strain in the microbial background community of the three different donors. Clostridium symbiosum (OTU56), was a unique feature of CGB treatment (Figure 2D).

When analyzing each individual independently, CG increased Rikenellaceae families 1 and 3, whereas in donor 3, Muribaculaceae, Prevotellaceae, and unclassified members of the class Bacteroidia were also enriched (Supplementary Table S5). Co-supplementation of the 
probiotic with chitin-glucan did not further stimulate Bacteroidetes phylum members in any of the donors.

At the mucosal level, different treatments had less effect than in the luminal compartment. At family level, no significant effect was observed (Adonis test based on BrayCurtis distance, $p=0.847$ ), although the DAPC plot shows a different clustering between treatments and control (Figure 3A). At OTU level, B. breve (OTU7), Butyrate-producing bacterium-L212 (OTU12) and Fusicatenibacter saccharivorans/Clostridium clostridioforme (OTU18) were enriched in CGB-treated reactors, (Figure 3B,C). Two unique features were found in the mucosal environment of the CGB treatment, corresponding to B. breve (OTU7) and B. bifidum (OTU21). Unclassified_Firmicutes (OTU91) was unique for the CG treatment, while Coprococcus catus (OTU58) was only detected in CG and CGB, but not in the control condition (Figure 3D).

A

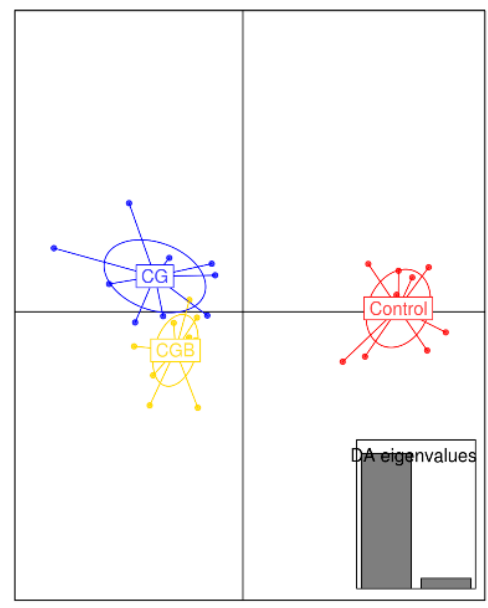

B

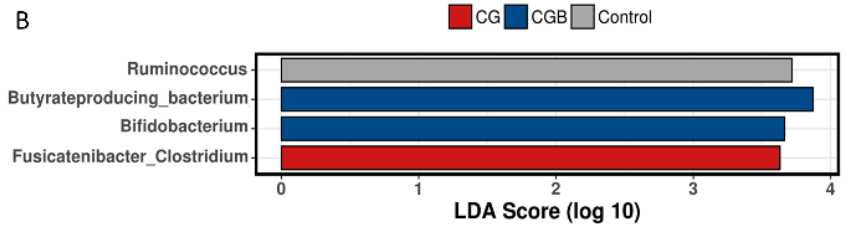

C
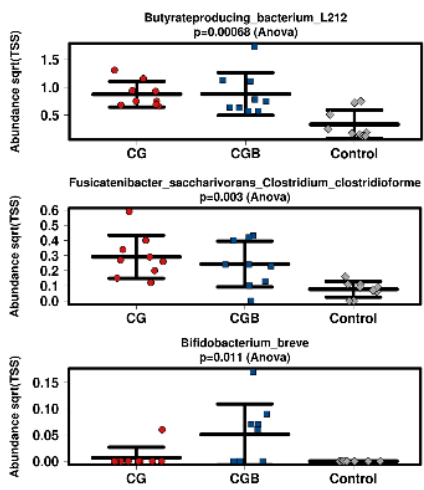

D

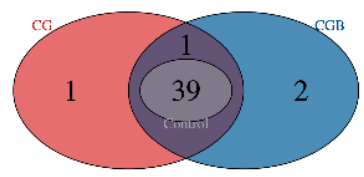

Figure 3. Effect of GC and GCB treatments on mucosal microbial composition. (A) Discriminant analysis of principal components (DAPC) plot of the mucosal compartment at family level; (B) core microbiota at OTU level; (C) linear discriminant analysis effect size (LEfSe) at family level; (D) selected significant different features at OTU level between control and treatments. Graphs represent $16 \mathrm{~S}$ rRNA sequencing data from three healthy individuals $(\mathrm{n}=3)$ in technical triplicates $(n=3)$.

Interindividual differences were also observed in the mucosal compartment with a mild stimulatory effect of CG on Bifidobacterium adolescentis/B. faecale (OTU34) in donor 3. In donors 1 and 2, CG stimulated Bacteroidaceae family, corresponding to Bacteroides uniformis (OTU8 and OTU11) (Supplementary Tables S6 and S7). OTU9, associated with a member of the Lachnospiraceae NK4A136 group, was enriched in donors 1 and 3, while Eubacteriaceae and Eryspelatoclostridiaceae members were stimulated in donors 2 and 1, respectively (Supplementary Tables S6 and S7).

\subsection{Specific Modulation of Bifidobacteria and F. prausnitzii by CG and CGB Based on qPCR Quantification}

The effect of different treatments on bifidobacteria and F. prausnitzii was donordependent. In the luminal compartment, CG-treated reactors showed a higher ratio of Bifidobacterium at $48 \mathrm{~h}$ compared to T0, with consistent results for the three donors (Figure 4A,B). At $24 \mathrm{~h}, \mathrm{CGB}$ increased the ratio of Bifidobacterium in all the donors, and only in donor 1 and donor 2 at $48 \mathrm{~h}$. Mucosal colonization with Bifidobacterium was only affected by CGB in donor 1 at $48 \mathrm{~h}$ (Figure 4C). 

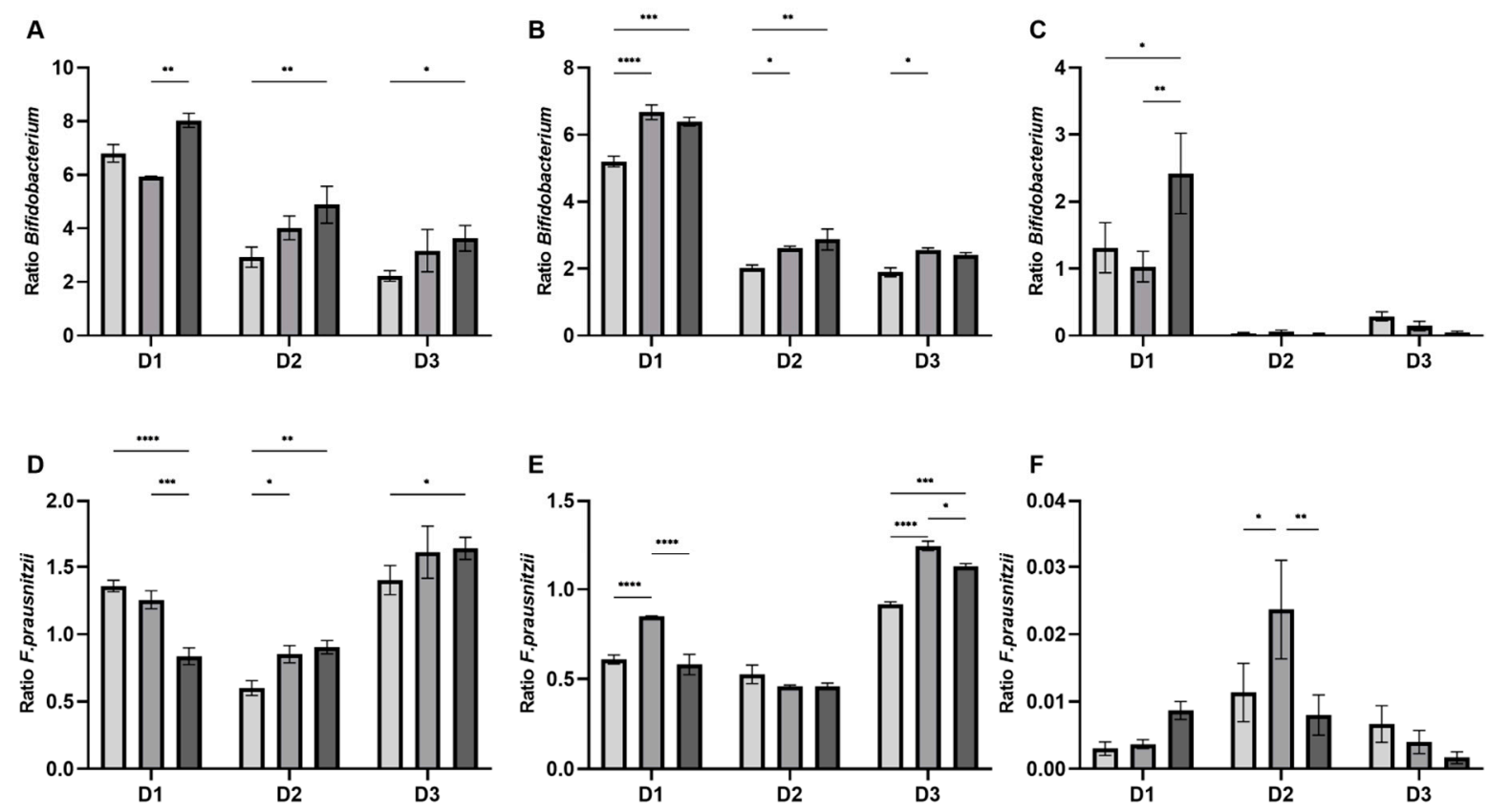

Figure 4. Effect of CG and CGB on specific members of the microbial community. Bars represent the ratio between bifidobacteria (A-C) or F. prausnitzii (D-F) qPCR counts (copies/mL) in control, CG and CGB conditions respect to time 0 levels. Each donor is represented in the $X$ axis $(D 1, D 2, D 3 ;$ mean $\pm S E M ; n=3)$. Graphs $(\mathbf{A}, \mathbf{D})$ and graphs (B,E) represent the luminal compartment at $24 \mathrm{~h}$ and $48 \mathrm{~h}$, respectively. Graphs $\mathrm{C}$ and $\mathrm{F}$ represent the mucosal compartment at $48 \mathrm{~h}$. Statistically significant differences were calculated using of bifidobacterial or $F$. prausnitzii between control and treatments (nutrients-1355053, two-way ANOVA) and are marked with asterisks ${ }^{*} p<0.05 ;{ }^{* *} p<0.01 ;{ }^{* * *} p<0.001$; $* * * * p<0.0001)$.

F. prausnitzii ratio in the luminal compartment at $24 \mathrm{~h}$ was increased in donor 2 by CG and CGB treatments and in donor 3 only by CGB, while in donor 1 , the opposite trend was observed for both conditions (Figure 4D). At $48 \mathrm{~h}$, donor 1 showed a different response, with increases in F. prausnitzii ratio by CG. Similarly, F. prausnitzii ratio was also increased in donor 3 by CG but also by CGB treatment (Figure 4E).

In the mucosal compartment, only donor 2 showed an increased F. prausnitzii ratio, despite the low levels $(<0.05)$ (Figure $4 \mathrm{~F})$.

3.4. Fecal Supernatants from CG and CGB Colonic Fermentations Improved Inflammation-Induced Damage to the Intestinal Epithelium and Modulate Cytokine Production

The effect of filter-sterilized samples from CG and CGB reactors on epithelial barrier function and modulation of cytokine production was tested in a gut inflammation model.

Cells exposed to colonic simulated supernatants supplemented with CG and CGB for 24 and $48 \mathrm{~h}$ showed higher TEER values than the control condition $(p<0.05)$. (Figure 5A).

Interindividual differences in TEER response are shown in Supplementary Figure S4. The highest response was observed in donor 3 after exposure to CG $(110.6 \pm 1.3 \%)$ and CGB $(113.1 \pm 1.9 \%) 48$ h supernatants.

In general, all colonic batch suspensions increased the NF- $\mathrm{kB}$ activity above the positive control (LPS). Concretely, cells exposed to $24 \mathrm{~h}$ and $48 \mathrm{~h}$ CG supernatants showed higher NF- $\mathrm{kB}$ activity values compared to the control condition (Figure 5B). GCB supernatants also induced an increase in NF- $\mathrm{kB}$ at $24 \mathrm{~h}$ and $48 \mathrm{~h}$ (Figure 5B). Interindividual differences in NF- $\mathrm{KB}$ response to CG and CGB supernatants are presented in Supplementary Figure S5. 

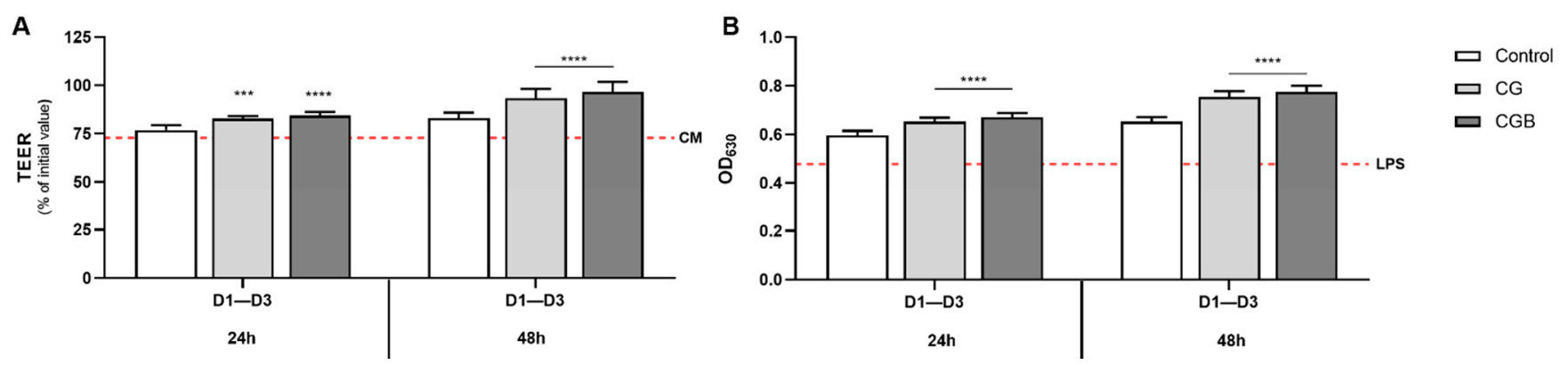

Figure 5. Effect of simulated colonic fluids exposed to CG and CGB during $24 \mathrm{~h}$ and $48 \mathrm{~h}$ on transepithelial electrical resistance (A) and NF- $\mathrm{kB}$ activation $(\mathbf{B})$ in a simulated colonic model. Bars represent the mean \pm SEM $(\mathrm{n}=9)$. Significant differences between control condition and different treatments are represented by $\left({ }^{* * *} p<0.001\right)$ and $(* * * p<0.0001)$. $\mathrm{CM}=$ cell culture media (negative control); LPS = lipopolysaccharide (positive control).

CG and CGB had a significant effect on the production of cytokines in the inflamed simulated epithelium (Figure 6). Compared to the LPS+ control, both CG and CGB $24 \mathrm{~h}$-colonic suspensions increased IL6, IL10 and IL1 $\beta$ levels compared to their controls $(p<0.05)$ (Figure 6A-C). For IL6 and IL10, this effect was most pronounced in cells exposed to $48 \mathrm{~h}$ colonic suspension (Figure 6A,B), without significant differences between CG and CGB treatments. For IL1 $\beta$, donor-specific responses were observed in cells exposed to $48 \mathrm{~h}$ colonic incubations (Supplementary Figure S6). TNF $\alpha$ levels were induced by $24 \mathrm{~h}$ CG colonic supernatants compared to control $(p<0.05)$, while 48 h CG colonic supernatants reduced TNF $\alpha$ levels (Figure $6 \mathrm{D}$ ). When considering all donors together, CGB did not show significant differences in TNF $\alpha$ levels; however, specific trends for each donor can be observed in Supplementary Figure S6. Concretely, CG treated samples from donor 3 significantly increased the TNF- $\alpha$ secretion compared to the control. Samples of $48 \mathrm{~h}$ of colonic fermentation of both CG and CGB reduced secretion of TNF- $\alpha$ in donors 1 and 3, while in donor 2, CGB significantly increased the TNF- $\alpha$ secretion.
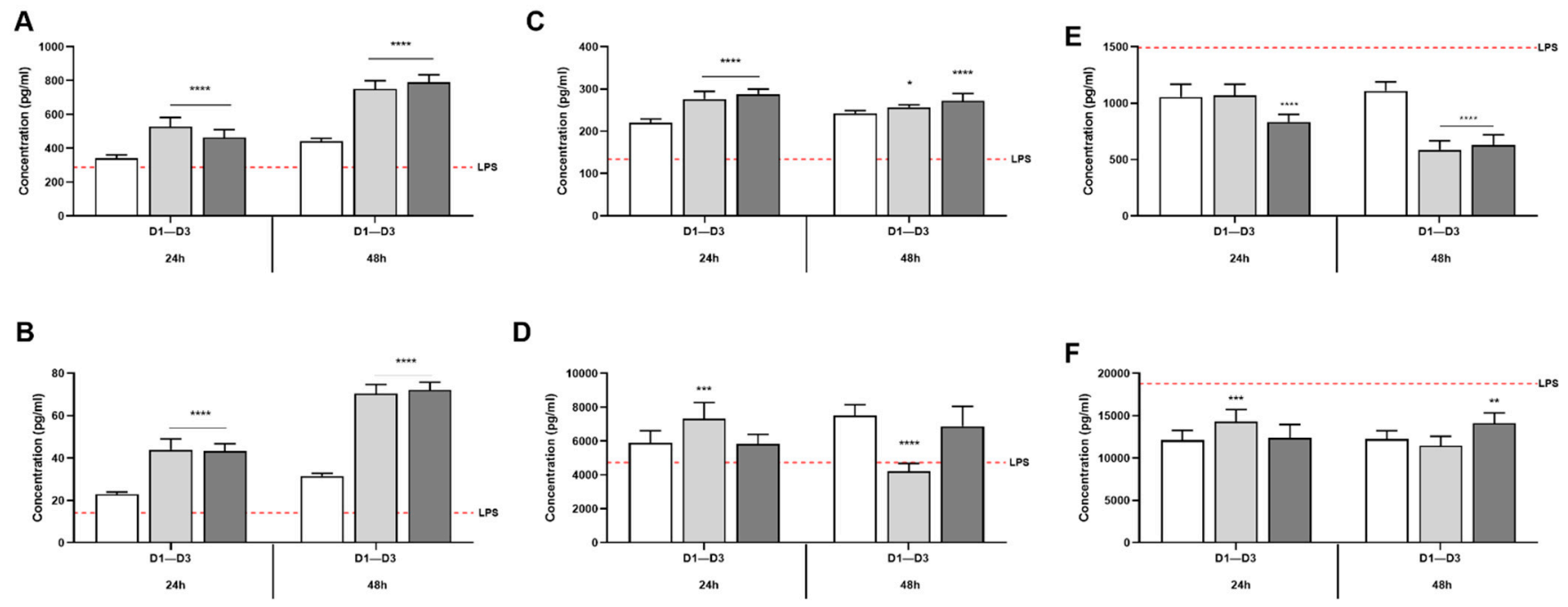

D
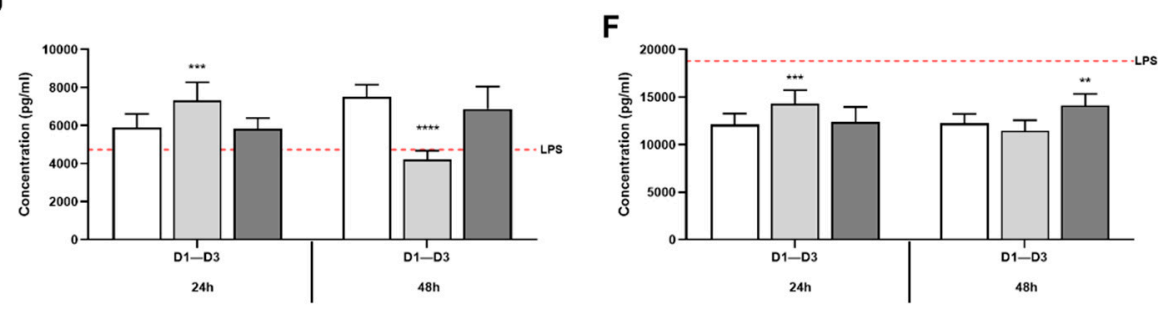

Figure 6. Effect of simulated colonic fluids exposed to CG and CGB during $24 \mathrm{~h}$ and $48 \mathrm{~h}$ on cytokine production. Bars represent the mean (pg/mL) \pm SEM, $\mathrm{n}=9$ of IL6 (A), IL10 (B), IL1ß (C), TNF $\alpha$ (D), CXCL10 (E), and MCP1 (F). Significant differences between control condition and different treatments are represented by $\left(^{*}\right) .\left({ }^{*}\right),\left(^{* *}\right),\left({ }^{* * *}\right)$, and $\left({ }^{* * *}\right)$ represent $p<0.05, p<0.01, p<0.001$, and $p<0.0001$, respectively. LPS = lipopolysaccharide (positive control).

CXCL10 levels were reduced by $24 \mathrm{~h}$ CGB supernatants compared to control condition $(p<0.05)$. After $48 \mathrm{~h}$ of fermentation, both CG and CGB supernatants reduced CXCL10 levels compared to the control $(p<0.05)$ (Figure 6E). The highest inhibitory effect on CXCL10 production was observed for donors 1 and 3 (Supplementary Figure S6). 
Overall production of MCP1 was increased by $24 \mathrm{~h}$ CG and 48 h CGB colonic supernatants compared to the control $(p<0.05)$ (Figure 6F). In donors 2 and 3, 24 h CGB treatment significantly decreased the MCP-1 secretion. After $48 \mathrm{~h}$ of colonic fermentation, both CG and CGB treatment significantly decreased MCP-1 secretion in donor 3 (Supplementary Figure S6).

\section{Discussion}

This study showed that CG and CGB supplementation modulate microbial activity, mucosal colonization and cytokine production in vitro, with consistent results across donors in improving the intestinal epithelial barrier, inducing anti-inflammatory IL10 production and promoting butyrate-producing bacteria colonization of the mucosal niche.

A single dose $(5 \mathrm{~g} / \mathrm{L})$ of CG and CGB increased total short-chain fatty acid production, with a consistent effect on the three tested donors. Concretely, health-promoting butyrate and propionate were stimulated by both treatments. These SCFAs are involved in regulating host energy metabolism and preserving mucosal integrity, with both intestinal and extra-intestinal effects $[36,37]$. Butyrate is a primary energy source for colonocytes and supports intestinal homeostasis through anti-inflammatory activity, while propionate modulates the secretion of GLP-1 and PYY by enteroendocrine cells in the gut [38]. Acetate also has a significant function on intestinal homeostasis, regulating gut $\mathrm{pH}$, acting as an energy substrate for colonocytes, having a significant impact on host metabolism, and playing a role in preventing intestinal infections [39,40]. Remarkably, inflammatory bowel disease (IBD) patients have lower SCFAs levels in feces than healthy controls, together with reduced levels of SCFAs-producing bacteria, including F. prausnitzii and Roseburia intestinalis, in intestinal mucosa and feces [41]. The effect of CG on increasing SCFAs may be beneficial for improving intestinal health in specific dysbiotic conditions. In that sense, shifts in microbial communities observed in our in vitro study are in line with results from human interventions and in vivo animal studies using CG [16,18]. Rodriguez et al., 2020 showed increases in Roseburia spp. after a 3 week intervention in humans with $4.5 \mathrm{~g}$ CG/day. In our study, changes in microbiota composition were already observed after $48 \mathrm{~h}$ of treatment, indicating a quick modulatory activity of CG towards healthy microbial ecosystems, with specific increase in Roseburia spp. related to $R$. hominis and $R$. inulinivorans.

We incorporated the mucosal environment in the in vitro setup to obtain a better understanding of prebiotic mechanisms at the host interface. The close interplay between gut epithelium and mucosal microbial communities has been described as a key factor for immune modulation, intestinal maturation, and competitive exclusion of pathogens [42-44]. The mucosal ecosystems have a different structure from the luminal milieu, including specific microorganism assembly $[43,44]$. We observed the colonization of the mucosal niche by B.breve and B. bifidum in CGB treated-reactors, the last potentially present as commensal in the fecal background microbiota. Schroeder et al., 2019 suggested that Bifidobacterium strains prevent intestinal disease by modulating the function of the mucus layer [45]. Concretely, several human disorders related to autoimmunity and dysregulated pro-inflammatory paths have been linked to a reduction in the abundance of mucosal $F$. prausnitzii and Coprococcus spp. [46]. From our results, Coprococcus spp. was only present in the mucosal communities of CG and CGB treatments and not in the control condition. Mucins also serve as a growth substrate for butyrate-producing bacteria, possibly via cross-feeding with mucin-degrading microbes such bifidobacteria that deliver partial breakdown products, acetate and/or lactate $[47,48]$. The main difference induced in the mucosal structure between CG and CGB was related to the presence of $B$. breve as a unique feature in CGB treatments, suggesting a minor impact of the probiotic strain compared to CG on background microbiota. Similarly, the effect of CG on microbial metabolism was not modified with the presence of $B$. breve, suggesting that the prebiotic activity of CG has a major role in modulating the microbial activity, while B. breve did not support a synergistic effect. 
Based on 16S RNA data, GC and GCB increased a butyrate-producing bacteriumL212 in luminal and mucosal compartments, while F. prausnitzii ratio was increased by both treatments, especially in the luminal compartment. F. prausnitzii has been previously described as an indicator of intestinal health and a key commensal microorganism with immunomodulatory properties $[49,50]$.

In that sense, we observed that filter-sterilized fecal supernatants from CG and CGB improved epithelial barrier damage in an inflammatory intestinal model, and increased IL10 and IL6 production while reducing proinflammatory TNF $\alpha$ and CXCL10. The impaired epithelial barrier has been associated with inflammatory pathological conditions of the gut and restoration of intestinal function has been proposed as a potential therapeutic target to promote recovery of intestinal homeostasis [51]. Recently, a microbial anti-inflammatory molecule (MAM) produced by F. prausnitzii efficiently re-established the intestinal barrier structure via the regulation of the tight junction pathway and ZO-1 expression [52], while previous studies also showed that F. prausnitzii is associated with anti-inflammatory effects via IL10 induction in dendritic cells [53]. Roseburia intestinalis and bifidobacteria have also been described as effective commensals in promoting intestinal barrier function [54-56]. In that sense, MAM has also been described as a down regulator of NFk $\beta$ [55], which, in our study, was increased by filter-sterilized supernatants derived from CG and CGB fermentations. Linked to cytokine production, NFk $\beta$ signaling is also responsible for coordinating inflammatory and immune responses, also participating in the control of cell proliferation and survival and maintaining epithelial barrier function [57]. In our model, NFk $\beta$ activation is enhanced upon butyrate treatment, linked to the expression of the immunomodulatory cytokines IL-6 and IL-10 [21]. CG and CGB similarly increase NFk $\beta$ activity, potentially modulating cytokine production. Microbial metabolites, such as butyrate, acetate, or propionate, and unknown molecules may also be involved in the response observed in our model. For example, butyrate has been shown to enhance IL-10 secretion in human monocytes. IL-10 is a central cytokine in maintaining mucosal tolerance, signaling different lymphocyte $\mathrm{T}$ subpopulations, and influencing selective colonization of the gut [58], and it has been proposed as a target for alleviating gut inflammatory conditions [59]. Moreover, Roseburia intestinalis derived flagellin has been proved as an effective modulator of inflammatory gut responses [60]. In addition, fecal supernatants derived from chitin-glucan fermentation had a stimulatory effect on IL1 $\beta$ production while reducing CXCL10, suggesting that they have a significant modulatory role in cytokine production. Further assessment including more donors and testing specific signaling pathways are needed.

Despite the current limitations of this study, such as the limited number of donors, the microbiota analysis based on the taxonomic profile via 16S rRNA gene sequencing, and lack of characterization of the metabolic activity in the mucosal niche, we proved a mechanistic link between CG and CGB, gut microbiota modulation, and intestinal health. The potential of CG and CGB for balancing the anti- and pro-inflammatory status and improving epithelial barrier deserves further investigation in human populations.

\section{Conclusions}

Using a combination of complex in vitro tools mimicking the gut microbiota from the mucosal and luminal environment and the host intestinal barrier, we demonstrated that CG and CGB supplementation could consistently stimulate Roseburia spp. and butyrate-producing bacteria while inducing a significant improvement of the inflammationdisrupted epithelial barrier and modulated cytokine production. B. breve colonized both, luminal and mucosal compartment. Incorporating the mucosal niche provides novel information on potential interactions between pre- and pro-biotic combinations and complex microbial communities.

Supplementary Materials: The following are available online at https: / /www.mdpi.com/article / 10.3390/nu13093249/s1, Table S1: Primers used to quantify bifidobacteria, and Faecalibacterium prausnitzii by qPCR. Table S2: Conditions used to quantify bifidobacterial and Faecalibacterium 
prausnitzii by qPCR. Table S3: Effect of CG and CGB on lactate change $(\Delta 6 \mathrm{~h})$ for three independent donors. Data represent mean \pm SEM of $n=3$ technical replicas. Statistically significant differences ( $p<0.05$, unpaired $t$-test) between treatments and control are marked in bold. Table S4: Effect of CG and CGB on BCFA change $(\Delta 48 \mathrm{~h})$ for three independent donors. Data represent mean \pm SEM of $\mathrm{n}=3$ technical replicas. Statistically significant differences $(p<0.05$, unpaired $t$-test) between treatments and control are marked in bold. Table S5: Average abundances (log (cells $/ \mathrm{mL}))$ of the bacterial families in the luminal environment in the different test conditions (Ctrl = control, $\mathrm{CG}=$ chitin-glucan, $\mathrm{CGB}=$ chitin-glucan + B. breve) after $48 \mathrm{~h}$. Statistically significant differences are indicated in bold. The intensity of shading is correlated with the abundance of a given family under different conditions per donor. Significant differences reported in the tables (bold values) refer to differences CG versus the control condition and to CG versus CGB. Table S6: Average relative abundances (\%) of the bacterial families in the mucosal environment in the different test conditions ( $\mathrm{Ctrl}=$ control, $\mathrm{CG}=$ chitin-glucan, $\mathrm{CGB}=$ chitin-glucan + B. breve $)$ after $48 \mathrm{~h}$. Statistically significant differences are indicated in bold. The intensity of shading is correlated with the abundance of a given family under different conditions per donor. Significant differences reported in the tables (bold values) refer to differences CG versus the control condition and to CG versus CGB. Table S7: Average relative abundances (\%) of the most abundant OTUs in the mucosal environment in the different test conditions ( $\mathrm{Ctrl}=$ control, $\mathrm{CG}=$ chitin-glucan, $\mathrm{CGB}=$ chitin-glucan + B. breve) after $48 \mathrm{~h}$. Statistically significant differences are indicated in bold. The intensity of shading is correlated with the abundance of a given family under different conditions per donor. Significant differences reported in the tables (bold values) refer to differences CG versus the control condition and to CG versus CGB. Figure S1: Effect of CG and CGB on SCFA and BCFA in different donors. Figure S2: Diversity, richness and evenness in the luminal compartment. Figure S3: Diversity, richness and evenness in the mucosal compartment. Figure S4: Interindividual differences of TEER in response to CG and CGB. Figure S5: Interindividual differences of $N F k \beta$ in response to CG and CGB. Figure S6: Interindividual differences of cytokine production in response to CG and CGB.

Author Contributions: Conceptualization: L.V., J.G., S.M., T.R. and V.M.; Data curation: L.V., J.G. and M.C.; Methodology: J.G. and L.V.; Project administration: M.M. and P.V.d.A.; Writing-original draft, M.C.; Writing-review and editing, M.C., L.V., S.M., T.R. and V.M. All authors have read and agreed to the published version of the manuscript.

Funding: This research received funding from the competitive cluster Wagralim (ADIPOSTOP project, convention 7366), Wallonia, Belgium.

Institutional Review Board Statement: The study was conducted according to the guidelines of the Declaration of Helsinki, and approved by the Ethical Committee University Hospital Ghent (reference number B670201836585).

Informed Consent Statement: Informed consent was obtained from all subjects involved in the study.

Data Availability Statement: Data supporting reported results can be obtained by direct request to corresponding author (M.M.)

Acknowledgments: Authors thank Jelle De Medts, Evi Steppé and Gilles De Rocker for their technical assistance.

Conflicts of Interest: M.C., L.V. and J.G. are employed by ProDigest BV. PVDA was employed by ProDigest during the time this research was performed. M.M is CEO of ProDigest. S.M., T.R. and V.M. are employed by KitoZyme, Herstal, Belgium. All authors participated in the scientific decisions and agreed in protocols and publication.

\section{References}

1. Durack, J.; Lynch, S.V. The gut microbiome: Relationships with disease and opportunities for therapy. J. Exp. Med. 2019, 216, 20-40. [CrossRef]

2. Makki, K.; Deehan, E.C.; Walter, J.; Bäckhed, F. The Impact of Dietary Fiber on Gut Microbiota in Host Health and Disease. Cell Host Microbe 2018, 23, 705-715. [CrossRef]

3. McCleary, B.; Cox, J.; Ivory, R.; Delaney, E. Definition and analysis of dietary fiber in grain products. In Cereal Grain-Based Functional Foods; Royal Society of Chemistry: Cambridge, UK, 2018; pp. 103-126.

4. McCleary, B.V. Total dietary fiber (CODEX definition) in foods and food ingredients by a rapid enzymatic-gravimetric method and liquid chromatography: Collaborative study, first action 2017.16. J. AOAC Int. 2019, 102, 196-207. [CrossRef] 
5. Cruz-Requena, M.; Escobedo-García, S.; Salas-Tovar, J.; Mora-Cura, Y.; Chávez-González, M.; Castillo-Reyes, F.; Flores-Gallegos, A.; Rodríguez-Herrera, R. Definitions and regulatory perspectives of dietary fibers. In Dietary Fiber: Properties, Recovery, and Applications; Elsevier: London, UK, 2019; pp. 1-25.

6. Stephen, A.M.; Champ, M.M.; Cloran, S.J.; Fleith, M.; van Lieshout, L.; Mejborn, H.; Burley, V.J. Dietary fibre in Europe: Current state of knowledge on definitions, sources, recommendations, intakes and relationships to health. Nutr. Res. Rev. 2017, 30, 149-190. [CrossRef]

7. Hill, C.; Guarner, F.; Reid, G.; Gibson, G.R.; Merenstein, D.J.; Pot, B.; Morelli, L.; Canani, R.B.; Flint, H.J.; Salminen, S.; et al. The International Scientific Association for Probiotics and Prebiotics consensus statement on the scope and appropriate use of the term probiotic. Nat. Rev. Gastroenterol. Hepatol. 2014, 11, 506-514. [CrossRef] [PubMed]

8. Swanson, K.S.; Gibson, G.R.; Hutkins, R.; Reimer, R.A.; Reid, G.; Verbeke, K.; Scott, K.P.; Holscher, H.D.; Azad, M.B.; Delzenne, N.M.; et al. The International Scientific Association for Probiotics and Prebiotics (ISAPP) consensus statement on the definition and scope of synbiotics. Nat. Rev. Gastroenterol. Hepatol. 2020, 17, 687-701. [CrossRef] [PubMed]

9. Quagliani, D.; Felt-Gunderson, P. Closing America's Fiber Intake Gap: Communication Strategies from a Food and Fiber Summit. Am. J. Lifestyle Med. 2016, 11, 80-85. [CrossRef] [PubMed]

10. Neyrinck, A.M.; Rodriguez, J.; Vinoy, S.; Maquet, V.; Walter, J.; Bischoff, S.C.; Laville, M.; Delzenne, N.M. The FiberTAG project: Tagging dietary fibre intake by measuring biomarkers related to the gut microbiota and their interest for health. Nutr. Bull. 2020, 45, 59-65. [CrossRef] [PubMed]

11. Myhrstad, M.C.W.; Tunsjø, H.; Charnock, C.; Telle-Hansen, V.H. Dietary Fiber, Gut Microbiota, and Metabolic RegulationCurrent Status in Human Randomized Trials. Nutrients 2020, 12, 859. [CrossRef] [PubMed]

12. EFSA Panel on Dietetic Products, Nutrition and Allergies (NDA). Scientific Opinion on Dietary Reference Values for carbohydrates and dietary fibre. EFSA J. 2010, 8, 1462. [CrossRef]

13. Davani-Davari, D.; Negahdaripour, M.; Karimzadeh, I.; Seifan, M.; Mohkam, M.; Masoumi, S.J.; Berenjian, A.; Ghasemi, Y. Prebiotics: Definition, Types, Sources, Mechanisms, and Clinical Applications. Foods 2019, 8, 92. [CrossRef]

14. EFSA Panel on Dietetic Products, Nutrition and Allergies (NDA). Scientific Opinion on the safety of 'Chitin-glucan' as a Novel Food ingredient. EFSA J. 2010, 8, 1687. [CrossRef]

15. Bays, H.; Evans, J.; Maki, K.; Evans, M.; Maquet, V.; Cooper, R.; Anderson, J. Chitin-glucan fiber effects on oxidized low-density lipoprotein: A randomized controlled trial. Eur. J. Clin. Nutr. 2013, 67, 2-7. [CrossRef]

16. Rodriguez, J.; Neyrinck, A.M.; Zhang, Z.; Seethaler, B.; Nazare, J.-A.; Robles Sánchez, C.; Roumain, M.; Muccioli, G.G.; Bindels, L.B.; Cani, P.D. Metabolite profiling reveals the interaction of chitin-glucan with the gut microbiota. Gut Microbes 2020, 12, 1810530. [CrossRef]

17. Marzorati, M.; Maquet, V.; Possemiers, S. Fate of chitin-glucan in the human gastrointestinal tract as studied in a dynamic gut simulator (SHIME®). J. Funct. Foods 2017, 30, 313-320. [CrossRef]

18. Neyrinck, A.M.; Possemiers, S.; Verstraete, W.; De Backer, F.; Cani, P.D.; Delzenne, N.M. Dietary modulation of clostridial cluster XIVa gut bacteria (Roseburia spp.) by chitin-glucan fiber improves host metabolic alterations induced by high-fat diet in mice. J. Nutr. Biochem. 2012, 23, 51-59. [CrossRef]

19. Ghouri, Y.A.; Richards, D.M.; Rahimi, E.F.; Krill, J.T.; Jelinek, K.A.; DuPont, A.W. Systematic review of randomized controlled trials of probiotics, prebiotics, and synbiotics in inflammatory bowel disease. Clin. Exp. Gastroenterol. 2014, 7, 473-487. [CrossRef] [PubMed]

20. Minami, J.; Iwabuchi, N.; Tanaka, M.; Yamauchi, K.; Xiao, J.-Z.; Abe, F.; Sakane, N. Effects of Bifidobacterium breve B-3 on body fat reductions in pre-obese adults: A randomized, double-blind, placebo-controlled trial. Biosci. Microbiota Food Health 2018, 37, 67-75. [CrossRef]

21. Van den Abbeele, P.; Taminiau, B.; Pinheiro, I.; Duysburgh, C.; Jacobs, H.; Pijls, L.; Marzorati, M. Arabinoxylo-oligosaccharides and inulin impact inter-individual variation on microbial metabolism and composition, which immunomodulates human cells. J. Agric. Food Chem. 2018, 66, 1121-1130. [CrossRef]

22. Van den Abbeele, P.; Belzer, C.; Goossens, M.; Kleerebezem, M.; De Vos, W.M.; Thas, O.; De Weirdt, R.; Kerckhof, F.-M.; Van de Wiele, T. Butyrate-producing Clostridium cluster XIVa species specifically colonize mucins in an in vitro gut model. ISME J. 2013, 7, 949-961. [CrossRef]

23. Pritchard, S.E.; Marciani, L.; Garsed, K.C.; Hoad, C.L.; Thongborisute, W.; Roberts, E.; Gowland, P.A.; Spiller, R.C. Fasting and postprandial volumes of the undisturbed colon: Normal values and changes in diarrhea-predominant irritable bowel syndrome measured using serial MRI. Neurogastroenterol. Motil. 2014, 26, 124-130. [CrossRef] [PubMed]

24. De Weirdt, R.; Possemiers, S.; Vermeulen, G.; Moerdijk-Poortvliet, T.C.; Boschker, H.T.; Verstraete, W.; Van de Wiele, T. Human faecal microbiota display variable patterns of glycerol metabolism. FEMS Microbiol. Ecol. 2010, 74, 601-611. [CrossRef] [PubMed]

25. De Paepe, K.; Kerckhof, F.M.; Verspreet, J.; Courtin, C.M.; Van de Wiele, T. Inter-individual differences determine the outcome of wheat bran colonization by the human gut microbiome. Environ. Microbiol. 2017, 19, 3251-3267. [CrossRef] [PubMed]

26. Kozich, J.J.; Westcott, S.L.; Baxter, N.T.; Highlander, S.K.; Schloss, P.D. Development of a dual-index sequencing strategy and curation pipeline for analyzing amplicon sequence data on the MiSeq Illumina sequencing platform. Appl. Environ. Microbiol. 2013, 79, 5112-5120. [CrossRef]

27. Schloss, P.D.; Westcott, S.L. Assessing and improving methods used in operational taxonomic unit-based approaches for 16S rRNA gene sequence analysis. Appl. Environ. Microbiol. 2011, 77, 3219-3226. [CrossRef] [PubMed] 
28. Schloss, P.D.; Westcott, S.L.; Ryabin, T.; Hall, J.R.; Hartmann, M.; Hollister, E.B.; Lesniewski, R.A.; Oakley, B.B.; Parks, D.H.; Robinson, C.J. Introducing mothur: Open-source, platform-independent, community-supported software for describing and comparing microbial communities. Appl. Environ. Microbiol. 2009, 75, 7537-7541. [CrossRef]

29. Wang, X.; Cai, Y.; Sun, Y.; Knight, R.; Mai, V. Secondary structure information does not improve OTU assignment for partial 16s rRNA sequences. ISME J. 2012, 6, 1277-1280. [CrossRef]

30. Wang, Q.; Garrity, G.M.; Tiedje, J.M.; Cole, J.R. Naïve Bayesian Classifier for Rapid Assignment of rRNA Sequences into the New Bacterial Taxonomy. Appl. Environ. Microbiol. 2007, 73, 5261-5267. [CrossRef]

31. Quast, C.; Pruesse, E.; Yilmaz, P.; Gerken, J.; Schweer, T.; Yarza, P.; Peplies, J.; Glöckner, F.O. The SILVA ribosomal RNA gene database project: Improved data processing and web-based tools. Nucleic Acids Res. 2012, 41, D590-D596. [CrossRef]

32. Cole, J.R.; Wang, Q.; Fish, J.A.; Chai, B.; McGarrell, D.M.; Sun, Y.; Brown, C.T.; Porras-Alfaro, A.; Kuske, C.R.; Tiedje, J.M. Ribosomal Database Project: Data and tools for high throughput rRNA analysis. Nucleic Acids Res. 2014, 42, D633-D642. [CrossRef]

33. Rinttilä, T.; Kassinen, A.; Malinen, E.; Krogius, L.; Palva, A. Development of an extensive set of 16S rDNA-targeted primers for quantification of pathogenic and indigenous bacteria in faecal samples by real-time PCR. J. Appl. Microbiol. 2004, 97, 1166-1177. [CrossRef]

34. Lopez-Siles, M.; Martinez-Medina, M.; Busquets, D.; Sabat-Mir, M.; Duncan, S.H.; Flint, H.J.; Aldeguer, X.; Garcia-Gil, L.J. Mucosa-associated Faecalibacterium prausnitzii and Escherichia coli co-abundance can distinguish Irritable Bowel Syndrome and Inflammatory Bowel Disease phenotypes. Int. J. Med. Microbiol. 2014, 304, 464-475. [CrossRef]

35. Daguet, D.; Pinheiro, I.; Verhelst, A.; Possemiers, S.; Marzorati, M. Arabinogalactan and fructooligosaccharides improve the gut barrier function in distinct areas of the colon in the Simulator of the Human Intestinal Microbial Ecosystem. J. Funct. Foods 2016, 20, 369-379. [CrossRef]

36. Chen, J.; Vitetta, L. The Role of Butyrate in Attenuating Pathobiont-Induced Hyperinflammation. Immune Netw. 2020, 20, e15. [CrossRef]

37. Zhang, L.; Liu, C.; Jiang, Q.; Yin, Y. Butyrate in Energy Metabolism: There Is Still More to Learn. Trends Endocrinol. Metab. 2021, 32, 159-169. [CrossRef]

38. Psichas, A.; Sleeth, M.L.; Murphy, K.G.; Brooks, L.; Bewick, G.A.; Hanyaloglu, A.C.; Ghatei, M.A.; Bloom, S.R.; Frost, G. The short chain fatty acid propionate stimulates GLP-1 and PYY secretion via free fatty acid receptor 2 in rodents. Int. J. Obes. 2015, 39, 424-429. [CrossRef]

39. Den Besten, G.; Van Eunen, K.; Groen, A.K.; Venema, K.; Reijngoud, D.-J.; Bakker, B.M. The role of short-chain fatty acids in the interplay between diet, gut microbiota, and host energy metabolism. J. Lipid Res. 2013, 54, 2325-2340. [CrossRef]

40. Fukuda, S.; Toh, H.; Hase, K.; Oshima, K.; Nakanishi, Y.; Yoshimura, K.; Tobe, T.; Clarke, J.M.; Topping, D.L.; Suzuki, T. Bifidobacteria can protect from enteropathogenic infection through production of acetate. Nature 2011, 469, 543-547. [CrossRef]

41. Venegas, D.P.; De la Fuente, M.K.; Landskron, G.; González, M.J.; Quera, R.; Dijkstra, G.; Harmsen, H.J.M.; Faber, K.N.; Hermoso, M.A. Short Chain Fatty Acids (SCFAs)-Mediated Gut Epithelial and Immune Regulation and Its Relevance for Inflammatory Bowel Diseases. Front. Immunol. 2019, 10, 277. [CrossRef]

42. Deng, Z.; Luo, X.M.; Liu, J.; Wang, H. Quorum Sensing, Biofilm, and Intestinal Mucosal Barrier: Involvement the Role of Probiotic. Front. Cell. Infect. Microbiol. 2020, 10. [CrossRef]

43. De Weirdt, R.; Van de Wiele, T. Micromanagement in the gut: Microenvironmental factors govern colon mucosal biofilm structure and functionality. NPJ Biofilms Microbiomes 2015, 1, 15026. [CrossRef] [PubMed]

44. Li, H.; Limenitakis, J.P.; Fuhrer, T.; Geuking, M.B.; Lawson, M.A.; Wyss, M.; Brugiroux, S.; Keller, I.; Macpherson, J.A.; Rupp, S.; et al. The outer mucus layer hosts a distinct intestinal microbial niche. Nat. Commun. 2015, 6, 8292. [CrossRef] [PubMed]

45. Schroeder, B.O. Fight them or feed them: How the intestinal mucus layer manages the gut microbiota. Gastroenterol. Rep. 2019, 7, 3-12. [CrossRef]

46. Bernstein, C.N.; Forbes, J.D. Gut microbiome in inflammatory bowel disease and other chronic immune-mediated inflammatory diseases. Inflamm. Intest. Dis. 2017, 2, 116-123. [CrossRef]

47. González-Rodríguez, I.; Ruiz, L.; Gueimonde, M.; Margolles, A.; Sánchez, B. Factors involved in the colonization and survival of bifidobacteria in the gastrointestinal tract. FEMS Microbiol. Lett. 2013, 340, 1-10. [CrossRef]

48. Belzer, C.; De Vos, W.M. Microbes inside-from diversity to function: The case of Akkermansia. ISME J. 2012, 6, 1449-1458. [CrossRef]

49. Miquel, S.; Martin, R.; Rossi, O.; Bermudez-Humaran, L.; Chatel, J.; Sokol, H.; Thomas, M.; Wells, J.; Langella, P. Faecalibacterium prausnitzii and human intestinal health. Curr. Opin. Microbiol. 2013, 16, 255-261. [CrossRef] [PubMed]

50. Sokol, H.; Pigneur, B.; Watterlot, L.; Lakhdari, O.; Bermúdez-Humarán, L.G.; Gratadoux, J.-J.; Blugeon, S.; Bridonneau, C.; Furet, J.-P.; Corthier, G. Faecalibacterium prausnitzii is an anti-inflammatory commensal bacterium identified by gut microbiota analysis of Crohn disease patients. Proc. Natl. Acad. Sci. USA 2008, 105, 16731-16736. [CrossRef] [PubMed]

51. Odenwald, M.A.; Turner, J.R. The intestinal epithelial barrier: A therapeutic target? Nat. Rev. Gastroenterol. Hepatol. 2017, 14, 9-21. [CrossRef] [PubMed] 
52. Xu, J.; Liang, R.; Zhang, W.; Tian, K.; Li, J.; Chen, X.; Yu, T.; Chen, Q. Faecalibacterium prausnitzii-derived microbial antiinflammatory molecule regulates intestinal integrity in diabetes mellitus mice via modulating tight junction protein expression. $J$. Diabetes 2020, 12, 224-236. [CrossRef] [PubMed]

53. Kiernan, M.G.; Coffey, J.C.; Sahebally, S.M.; Tibbitts, P.; Lyons, E.M.; O'leary, E.; Owolabi, F.; Dunne, C.P. Systemic Molecular Mediators of Inflammation Differentiate Between Crohn's Disease and Ulcerative Colitis, Implicating Threshold Levels of IL-10 and Relative Ratios of Pro-inflammatory Cytokines in Therapy. J. Crohn Colitis 2019, 14, 118-129. [CrossRef]

54. Ling, X.; Linglong, P.; Weixia, D.; Hong, W. Protective effects of bifidobacterium on intestinal barrier function in LPS-induced enterocyte barrier injury of Caco-2 monolayers and in a rat NEC model. PLoS ONE 2016, 11, e0161635. [CrossRef]

55. Hiippala, K.; Jouhten, H.; Ronkainen, A.; Hartikainen, A.; Kainulainen, V.; Jalanka, J.; Satokari, R. The potential of gut commensals in reinforcing intestinal barrier function and alleviating inflammation. Nutrients 2018, 10, 988. [CrossRef]

56. Tan, B.; Luo, W.; Shen, Z.; Xiao, M.; Wu, S.; Meng, X.; Wu, X.; Yang, Z.; Tian, L.; Wang, X. Roseburia intestinalis inhibits oncostatin $\mathrm{M}$ and maintains tight junction integrity in a murine model of acute experimental colitis. Scand. J. Gastroenterol. 2019, 54, 432-440. [CrossRef] [PubMed]

57. Guma, M.; Stepniak, D.; Shaked, H.; Spehlmann, M.E.; Shenouda, S.; Cheroutre, H.; Vicente-Suarez, I.; Eckmann, L.; Kagnoff, M.F.; Karin, M. Constitutive intestinal NF- $\mathrm{kB}$ does not trigger destructive inflammation unless accompanied by MAPK activation. J. Exp. Med. 2011, 208, 1889-1900. [CrossRef]

58. Zhou, L.; Sonnenberg, G.F. Essential immunologic orchestrators of intestinal homeostasis. Sci. Immunol. 2018, 3, eaao1605. [CrossRef] [PubMed]

59. Wang, S.; Wang, J.; Ma, R.; Yang, S.; Fan, T.; Cao, J.; Wang, Y.; Ma, W.; Yang, W.; Wang, F. IL-10 enhances T cell survival and is associated with faster relapse in patients with inactive ulcerative colitis. Mol. Immunol. 2020, 121, 92-98. [CrossRef] [PubMed]

60. Quan, Y.; Song, K.; Zhang, Y.; Zhu, C.; Shen, Z.; Wu, S.; Luo, W.; Tan, B.; Yang, Z.; Wang, X. Roseburia intestinalis-derived flagellin is a negative regulator of intestinal inflammation. Biochem. Biophys. Res. Commun. 2018, 501, 791-799. [CrossRef] 